\title{
MAFLD Criteria May Overlook a Subtype of Patient with Steatohepatitis and Significant Fibrosis
}

\author{
Jiaofeng Huang $\mathbb{D}^{1, *}$ \\ Wenjuan Xue $\mathbb{B}^{2, *}$ \\ Mingfang Wang $\mathbb{D}^{1}$ \\ Yinlian Wu $\mathbb{D}^{\prime}$ \\ Medha Singh $\mathbb{D}^{3}$ \\ Yueyong Zhu (iD) ${ }^{1,4}$ \\ Rahul Kumar $\mathbb{D}^{5}$ \\ Su Lin (D) ${ }^{\prime}$
}

'Department of Hepatology, Hepatology Research Institute, The First Affiliated Hospital, Fujian Medical University, Fuzhou, Fujian, 350005, People's Republic of China; ${ }^{2}$ Department of Traditional Chinese Medicine, The First Affiliated Hospital of Fujian Medical University, Fuzhou, Fujian, 350005, People's Republic of China; ${ }^{3}$ Department of General Medicine, Tan Tock Seng Hospital, Singapore; ${ }^{4}$ Fujian Key Laboratory of Precison Medicine for Cancer, the First Affiliated Hospital, Fujian Medical University, Fuzhou, Fujian, People's Republic of China; ${ }^{5}$ Department of Gastroenterology and Hepatology, DukeNUS Academic Medical Centre, Changi General Hospital, Singapore

*These authors contributed equally to this work

Correspondence: Su Lin

Department of Hepatology, Hepatology Research Institute, The First Affiliated Hospital, Fujian Medical University, No. 20, Chazhong Road, Taijiang District, Fuzhou, Fujian, 350005, People's Republic of China

Tel +86 59I-8798I657

Fax +86 59I-87982526

Email sumer5I29@fjmu.edu.cn
Introduction: Metabolic associated fatty liver disease (MAFLD) is a novel concept for fatty liver disease. Different from non-alcoholic fatty liver disease (NAFLD), the diagnosis of MAFLD requires the presence of metabolic risks. This study aimed to characterize patients with liver steatosis but without metabolic risks (non-MR-steatosis) which may not be diagnosed by MAFLD criteria.

Methods: Consecutive patients who underwent biopsy were included in this study. The clinic-pathological characteristics of non-MR-steatosis, NAFLD and MAFLD were compared.

Results: A total of 1217 cases were included. There were 426 (35.00\%) cases with MAFLD, $585(48.07 \%)$ with NAFLD and $168(13.80 \%)$ with non-MR-steatosis. The majority of the cases were infected with HBV (93.26\%). The age and metabolic profiles were highest in MAFLD and lowest in non-MR-steatosis. The body mass index (BMI) level was also lowest in non-MR-steatosis $\left(20.78 \pm 1.54 \mathrm{~kg} / \mathrm{m}^{2}\right)$. The ALT and AST levels of the non-MR-steatosis group were not statistically different from those of MAFLD or NAFLD groups $(p>0.05)$. Histologically, there was no significant difference in the degrees of inflammation and fibrosis among the three groups. The severity of steatosis in non-MR-steatosis group was lower than MAFLD or NAFLD groups $(\mathrm{p}<0.05)$. These results were consistent in both HBV and nonHBV subgroups.

Conclusion: MAFLD criteria may overlook some steatotic patients without metabolic risks, who may also have steatohepatitis and significant fibrosis.

Keywords: metabolic associated fatty liver disease, nonalcoholic fatty liver disease, metabolic risks, lean NAFLD, biopsy

\section{Introduction}

Metabolic associated fatty liver disease (MAFLD) is a novel concept proposed by an international consensus in $2020 .^{1}$ Significantly different from non-alcoholic fatty liver disease (NAFLD), the diagnosis of MAFLD does not require the exclusion of other chronic liver diseases, while the presence of metabolic abnormality is necessary. ${ }^{2-5}$ Studies show the new definition of MAFLD helps to select patients at high intra- and extra-hepatic risk. ${ }^{5-8}$

The mortality in fatty liver disease is correlated with the severity of liver fibrosis. ${ }^{9,10}$ Our previous data also showed that MAFLD was more practical for identifying fatty liver disease with advanced fibrosis. ${ }^{11}$ On the other hand, we also found a group of patients with significant hepatic steatosis but without metabolic risks cannot be classified as MAFLD, may thus be undiagnosed. This group of patients could be labeled as non-metabolic related steatosis (non-MR-steatosis). ${ }^{12}$ 
However, the above two studies on the relationship between liver fibrosis and MAFLD ${ }^{11,12}$ were assessed by non-invasive fibrosis scores, which is suboptimal and inaccurate in MAFLD. ${ }^{13}$ There is a paucity of literature and a knowledge gap in this area as the severity of liver fibrosis is not assessed by the gold standard of liver biopsy. This study aimed to further evaluate the MAFLD criteria in a biopsy-proven cohort and to characterize patients who were currently undiagnosed by MAFLD criteria.

\section{Materials and Methods}

\section{Study Population}

This is a retrospective study designed to compare the differences between the MAFLD and NAFLD criteria in a liver biopsy cohort. Consecutive patients who underwent liver biopsy at the First Affiliated Hospital of Fujian Medical University between May 2005 and June 2015 were included. The study protocol was approved by the ethics committee of the First Affiliated Hospital of Fujian Medical University.

The inclusion criteria were all patients with biopsy during the study period. The exclusion criteria were as follows: 1) with any cause of acute hepatitis, for instance, acute viral hepatitis or drug-induced hepatitis; 2) autoimmune liver disease; 3) malignancy; 4) with incomplete data of relevant parameters.

This study cohort did not include any patient with excessive alcohol consumption as pathology is not necessary for the diagnosis and management of the alcoholic liver disease.

\section{Diagnostic Criteria and Definition of Groups MAFLD}

MAFLD is diagnosed based on biopsy-proven hepatic steatosis in the presence of any one of the following three metabolic conditions: diabetes mellitus, overweight/ obesity, or metabolic dysregulation. ${ }^{5}$ Overweight/obesity is defined as the body mass index (BMI) $\geq 23 \mathrm{~kg} / \mathrm{m}^{2}$. The type 2 diabetes mellitus was diagnosed based on a history of diabetes, and/or fasting plasma glucose $\geq 7.0 \mathrm{mmol} / \mathrm{L}$, or 2-hour glucose $\geq 11.1 \mathrm{mmol} / \mathrm{L}$, and/or glycosylated hemoglobin $\geq 6.5 \%$. According to the diagnosis criteria of MAFLD, ${ }^{5}$ metabolic dysregulation was defined when at least two metabolic abnormalities were presented, including the abnormal waist circumference, high blood pressure, low high-density lipoprotein cholesterol (HDL$\mathrm{C})$, high blood glucose or homeostasis model assessmentinsulin resistance (HOMA-IR) score and high C-reactive protein (CRP).

\section{NAFLD}

NAFLD was defined by the presence and pattern of fat accumulation (steatosis) on liver biopsy and by the exclusion of alcohol consumption of $\geq 30 \mathrm{~g} / \mathrm{d}$ for males and $\geq 20 \mathrm{~g} / \mathrm{d}$ for females or other known causes of liver disease. ${ }^{3}$ As the concomitance of HBV and NAFLD is popular in China ${ }^{14}$ and hepatitis $\mathrm{B}$ virus (HBV) infection rate was high in this cohort, we did not exclude those with HBV infection when diagnosing NAFLD, but performed a subgroup study in patients with and without HBV infection to exclude the impact of HBV infection in this study.

\section{Non-MR-Steatosis}

Patients with liver steatosis on liver biopsy but without obesity, metabolic dysregulation or type 2 diabetes were defined as non-MR-steatosis.

\section{HBV Infection}

The seropositive in hepatitis B surface antigen (HBsAg) for more than 6 months. ${ }^{15}$

\section{Demographic Variables}

The following demographic variables were obtained from the original database: age, sex, BMI, and the history of hypertension and type 2 diabetes mellitus. BMI was calculated as weight (in kilograms) divided by the square of height (in meters).

\section{Laboratory Parameters}

Laboratory measurement studied included fasting plasma glucose (FPG), total bilirubin (TBIL), aspartate aminotransferase (AST), alanine transaminase (ALT), $\gamma$-glutamyl transferase (GGT), albumin, blood urea nitrogen (BUN), uric acid, total cholesterol (TC), total triglyceride (TG), lowdensity lipoprotein cholesterol (LDL-C), HDL-C, very lowdensity lipoprotein cholesterol (VLDL-C), Hepatitis B e antigen (HBeAg) and HBV DNA. All biochemical assessments were performed by standard laboratory methods.

\section{Histologic Evaluation}

All patients enrolled in this study underwent ultrasonography guided percutaneous liver biopsy using a 16-gauge hepatic needle. The liver specimens were immediately immersed in formalin, embedded with paraffin, and stained with 
hematoxylin and eosin, and Masson's trichrome. A biopsy tissue with a minimum length of $15 \mathrm{~mm}$ and at least 6 portal areas was regarded as sufficient tissue. The liver biopsy specimen was reviewed by two experienced pathologists independently. Fatty liver was defined as the presence of steatosis in at least $5 \%$ of hepatocytes. Mild steatosis (S1) was defined if 5 $33 \%$, moderate steatosis (S2) if $>33-66 \%$, and severe steatosis (S3) if $>66 \%$ of examined liver surface area was involved with steatosis. ${ }^{16}$ The degree of liver inflammation was graded from 0 to 4 points use the Scheuer System. ${ }^{17,18}$ Liver fibrosis was scored from 0 to $4 .{ }^{17,18}$ Advanced fibrosis was defined as fibrosis score $\geq 3$ (fibrosis with architectural distortion). Significant inflammation was classified as inflammation grade $\geq 3 .{ }^{19}$ Moderate to severe steatosis was defined as steatosis grade $\geq 2$. NAFLD activity score (NAS) calculates the unweighted sum of the scores for steatosis $(0-3)$, lobular inflammation $(0-3)$ and hepatocellular ballooning $(0-2){ }^{16}$

\section{Statistical Analysis}

Continuous variables are expressed as means \pm standard variation (SD) or median (range interquartile). Categorical variables are expressed as percentages. The MannWhitney $U$-test (for 2 samples) or the Kruskal-Wallis for One-way Analysis of Variance (for K samples) and Chisquared test (for categorical variables) were used to investigate the differences between the groups. A multivariate logistic regression analysis was performed to explore the risk factors for advanced liver fibrosis. All tests were twotailed and resulted in a p-value less than 0.05 were considered statistically significant. All analysis was conducted using R 3.6.2 (https://www.r-project.org/) or SPSS 19.0.

\section{Results}

\section{Baseline Characteristics of Patients}

The consort diagram of population selection for the study is shown in Figure 1. A total of 1217 patients with liver biopsy and complete laboratory data were included. There were 966 (79.38\%) males, with a mean age of $37.58 \pm 11.28$ years, and a mean BMI of $22.97 \pm 3.23 \mathrm{~kg} / \mathrm{m}^{2}$. There were 107 (8.79\%) cases with type 2 diabetes and 61 (5.01\%) with hypertension. The majority of the cases $(1135 / 1217,93.26 \%)$ were HBsAg seropositive. All of the HBV-infected patients had never received anti-virus treatment before biopsy. Amongst the entire cohort, MAFLD was diagnosed in 426/1217 (35.00\%) participants, and NAFLD was diagnosed in 585/ 1217 (48.07\%). Significant inflammation (grade $\geq 3$ ) was found in 609 (50.04\%) patients, advanced fibrosis (scores $\geq 3)$ in $534(43.88 \%)$ patients, and moderate to severe steatosis (grade $\geq 2$ ) in $269(22.10 \%)$ patients.

A total of $168 / 1217(13.80 \%)$ patients had biopsy proven to have hepatic steatosis but without any metabolic

1217 subjects with liver biopsy and complete data from 2005- 2015

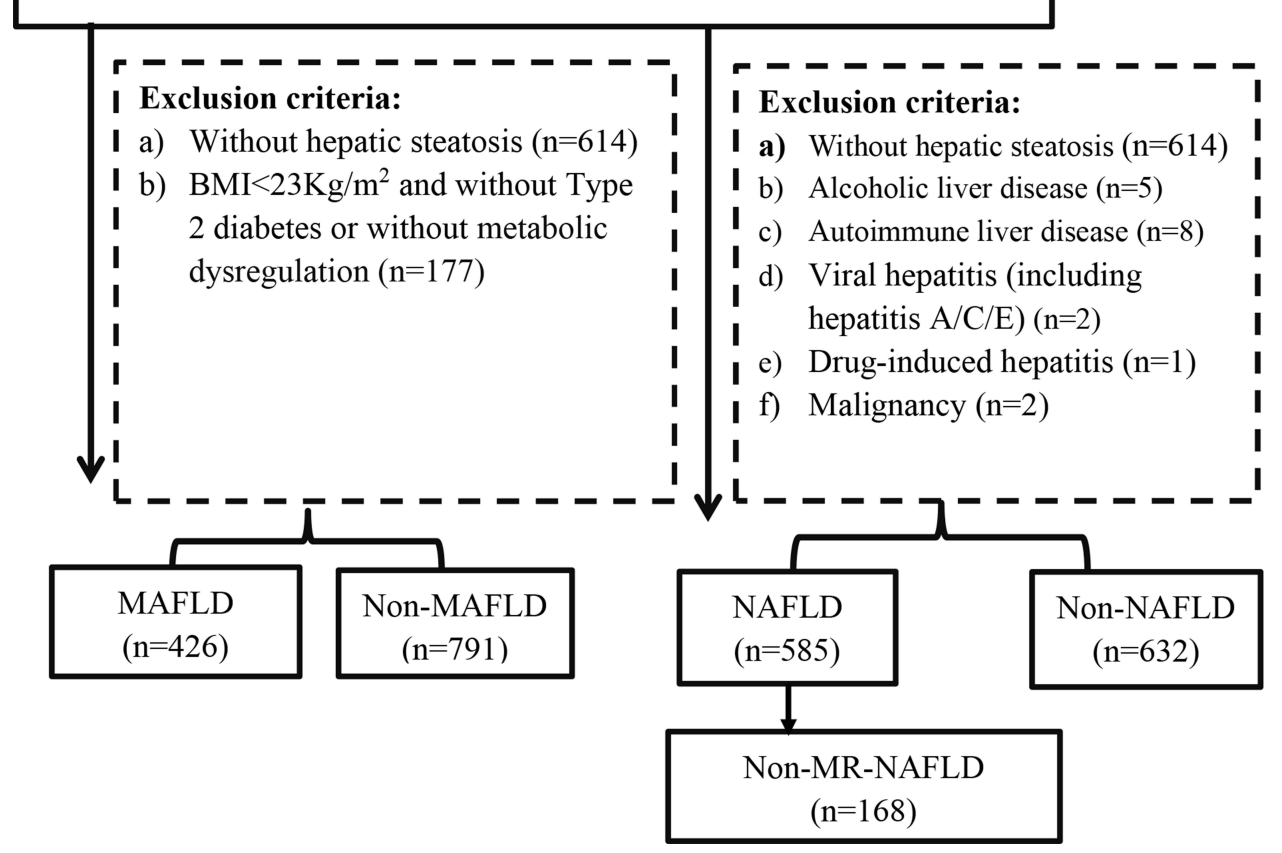

Figure I The flow chart. 
risks. These patients were grouped as non-MR-steatosis. Among these 168 patients, 150 (12.24\%) were missed diagnosed by both MAFLD. 74/1217 (6.08\%) patients were with NAFLD and without HBV infection.

\section{Comparison of MAFLD, NAFLD and Non-MR-Steatosis Groups}

The comparison among MAFLD, NAFLD and non-MRsteatosis is illustrated in Table 1. BMI of NAFLD group $\left(24.17 \pm 3.18 \mathrm{~kg} / \mathrm{m}^{2}\right)$ was significantly lower than that of MAFLD group $\left(25.51 \pm 2.66 \mathrm{~kg} / \mathrm{m}^{2}\right)$. The other metabolic profiles, including type 2 diabetes, TG, VLDL-C and FPG, were found to be higher in MAFLD population than NAFLD population. However, the difference between liver enzymes, HBeAg and HBV DNA was not statistically significant between NAFLD and MAFLD groups ( $\mathrm{P}>0.05$ ). The proportion of moderate to severe steatosis was $50.70 \%$ in the MAFLD group and $44.44 \%$ in the NAFLD group, but the difference did not reach statistical significance $(P=0.057)$. Similarly, there was no significant difference in inflammation and fibrosis between these two groups.

Patients in the non-MR-steatosis group had very different anthropometric and clinical characteristics compared to MAFLD and NAFLD groups: they were the youngest $(37.05 \pm 11.21$ years) among the three groups and had the lowest mean BMI level $\left(20.78 \pm 1.54 \mathrm{~kg} / \mathrm{m}^{2}\right)$. None of these patients had type 2 diabetes and only 4 $(2.38 \%)$ patients had hypertension. The serum lipids and the serum glucose-related indexes were all within normal range and were significantly lower than those of the MAFLD or NAFLD groups. The median serum ALT and AST levels of the non-MR-steatosis group were not different from the NAFLD and MAFLD groups $(\mathrm{P}>0.05)$. Histologically, there was no significant difference among the three groups in inflammation and advanced fibrosis, while the grade of steatosis in non-MR-steatosis group was lower than the MAFLD or NAFLD groups.

\section{The Comparison of MAFLD, NAFLD, and Overlap Group}

A total of 417 patients met the criteria of both MAFLD and NAFLD (overlap group). The comparison between MAFLD, NAFLD and the overlap group are shown in Supplementary Table 1. Overall, the features of overlap group were more similar to those of MAFLD. The overlap group had significantly higher BMI, blood lipids, and blood glucose than NAFLD. As for liver histopathology, no significant difference was found among the three groups. The multivariate logistic regression showed that males, higher ALT level and significant inflammation were independent risk factors for liver fibrosis $(\mathrm{P}<0.05$, detailed in Supplementary Table 2). However, the severity of hepatic steatosis was not independently associated with advanced fibrosis $(\mathrm{P}>0.05)$.

\section{Comparison Among MAFLD, NAFLD and Non-MR-Steatosis Groups in the Non-HBV Population}

Table 2 shows the characteristics of 82 cases without HBV infection. Among these patients, 60/82 (73.17\%) were diagnosed as MAFLD, 74/82 (90.24\%) as NAFLD, and $18 / 82(21.95 \%)$ as non-MR-steatosis. These three groups were significantly different in BMI $\left(25.3 \pm 3.35 \mathrm{~kg} / \mathrm{m}^{2}\right.$, $24.20 \pm 3.38 \mathrm{~kg} / \mathrm{m}^{2}$ and $20.97 \pm 1.25 \mathrm{~kg} / \mathrm{m}^{2}$, respectively, $\mathrm{P}<0.001$ ). Non-MR-steatosis group had significantly lower VLDL-C and FPG levels than NAFLD and MAFLD groups, however, other indicators including liver function and kidney function did not differ significantly among the three groups $(\mathrm{P}>0.05)$. The proportion of significant inflammation, advanced fibrosis, and moderate to severe steatosis in non-MR-steatosis group was $11.11 \%, 11.11 \%$ and $66.67 \%$, respectively, which was not different from the NAFLD and MAFLD group. As for NAS scores, there was no significant difference between the three groups.

\section{Comparison Among MAFLD, NAFLD and Non-MR-Steatosis Groups in the HBV-Infected Population}

Table 3 shows the characteristics of 1135 patients with HBV infection. Patients in the non-MR-steatosis group showed less metabolic profiles such as BMI, type 2 diabetes, blood lipids and blood glucose. The HBeAg positivity and HBV DNA level of the non-MR-steatosis group was not much different from those of MAFLD or NAFLD groups. The proportion of severe steatosis in the non-MRsteatosis group was lower than the other two groups while the inflammation and fibrosis were not significantly different among the three groups.

\section{Discussion}

The concept "MAFLD" is expected to change the management of fatty liver disease. ${ }^{4}$ This study compares the clinicopathological characteristics of MAFLD and 
Table I Comparison Between MAFLD, NAFLD and Non-MR-Steatosis

\begin{tabular}{|c|c|c|c|c|c|c|}
\hline \multirow[t]{2}{*}{ Variables } & \multirow[t]{2}{*}{ MAFLD } & \multirow[t]{2}{*}{ NAFLD } & \multirow{2}{*}{$\begin{array}{l}\text { Non-MR- } \\
\text { Steatosis }\end{array}$} & \multicolumn{3}{|c|}{$P$} \\
\hline & & & & $\begin{array}{c}\text { MAFLD } \\
\text { vs } \\
\text { NAFLD }\end{array}$ & $\begin{array}{l}\text { MAFLD vs } \\
\text { Non-MR- } \\
\text { Steatosis }\end{array}$ & $\begin{array}{c}\text { NAFLD vs } \\
\text { Non-MR- } \\
\text { Steatosis }\end{array}$ \\
\hline $\mathrm{N}$ & 426 & 585 & 168 & & & \\
\hline Age (years) & $40.39 \pm 10.97$ & $39.41 \pm 11.10$ & $37.05 \pm 11.21$ & 0.155 & 0.001 & 0.011 \\
\hline Male, n (\%) & 361 (84.74) & 489 (83.59) & $133(79.17)$ & 0.684 & 0.130 & 0.223 \\
\hline BMI $\left(\mathrm{kg} / \mathrm{m}^{2}\right)$ & $25.51 \pm 2.66$ & $24.17 \pm 3.18$ & $20.78 \pm 1.54$ & $<0.001$ & $<0.001$ & $<0.001$ \\
\hline Waist circumference $(\mathrm{cm})$ & $84.10 \pm 7.32$ & $84.69 \pm 6.82$ & $86.10 \pm 5.53$ & 0.740 & 0.445 & 0.572 \\
\hline Type 2 diabetes, $\mathrm{n}(\%)$ & $83(19.48)$ & $81(13.85)$ & $0(0)$ & 0.021 & $<0.001$ & $<0.001$ \\
\hline Hypertension, n (\%) & $42(9.86)$ & $45(7.69)$ & $4(2.38)$ & 0.272 & 0.004 & 0.022 \\
\hline HBsAg positive, n (\%) & $366(85.92)$ & $511(87.35)$ & I 50 (89.29) & 0.568 & 0.337 & 0.588 \\
\hline Log HBVDNA (IU/mL) & $4.88 \pm 2.24$ & $4.97 \pm 2.20$ & $5.11 \pm 2.13$ & 0.536 & 0.292 & 0.515 \\
\hline $\mathrm{HBeAg}$ positive, $n(\%)$ & $208(48.83)$ & $302(51.62)$ & $97(57.74)$ & 0.425 & 0.067 & 0.182 \\
\hline TBIL ( $\mu \mathrm{molL})$ & $18.44 \pm 21.50$ & $18.15 \pm 21.4$ & $17.4 \pm 21.02$ & 0.916 & 0.683 & 0.725 \\
\hline Albumin (g/L) & $42.58 \pm 4.95$ & $42.52 \pm 5.05$ & $42.25 \pm 5.24$ & 0.759 & 0.299 & 0.389 \\
\hline ALT (U/L) & $65(4 \mathrm{I}, 144)$ & $67(4|| 4 \mid .75)$, & $75.5(40,138.75)$ & 0.901 & 0.713 & 0.771 \\
\hline AST (U/L) & $42(30,7$ II) & $43(30,77.75)$ & $45(32,82.25)$ & 0.730 & 0.291 & 0.400 \\
\hline GGT (U/L) & $53(31,100)$ & $49(29,91)$ & $43(27,74)$ & 0.133 & 0.001 & 0.024 \\
\hline Creatinine $(\mu \mathrm{molL})$ & $73.35 \pm 13.82$ & $73.77 \pm 17.99$ & $74.19 \pm 26.07$ & 0.927 & 0.789 & 0.729 \\
\hline eGFR (mL/min) & $120.87 \pm 32.47$ & $121.83 \pm 37.01$ & $125.86 \pm 47.02$ & 0.875 & 0.364 & 0.404 \\
\hline Uric acid ( $\mu \mathrm{molL})$ & $369.22 \pm 82.29$ & $362.59 \pm 83.01$ & $345.03 \pm 81.38$ & 0.239 & 0.004 & 0.031 \\
\hline $\mathrm{TC}(\mathrm{mmol} / \mathrm{L})$ & $4.77 \pm 1.13$ & $4.74 \pm 1.08$ & $4.63 \pm 0.87$ & 0.749 & 0.312 & $0.41 \mathrm{I}$ \\
\hline TG (mmol/L) & $1.68 \pm 1.24$ & $1.50 \pm 1.11$ & $1.07 \pm 0.43$ & $<0.001$ & $<0.001$ & $<0.001$ \\
\hline LDL-C (mmol/L) & $2.90 \pm 0.97$ & $2.84 \pm 0.94$ & $2.69 \pm 0.82$ & 0.457 & 0.014 & 0.117 \\
\hline HDL-C (mmol/L) & $1.12 \pm 0.30$ & $1.19 \pm 0.34$ & $1.36 \pm 0.38$ & 0.002 & 0.043 & $<0.001$ \\
\hline VLDL-C (mmol/L) & $0.51 \pm 0.38$ & $0.46 \pm 0.35$ & $0.29 \pm 0.15$ & 0.003 & $<0.001$ & $<0.001$ \\
\hline FPG (mmol/L) & $5.34 \pm 1.42$ & $5.14 \pm 1.23$ & $4.71 \pm 0.54$ & 0.005 & $<0.001$ & $<0.001$ \\
\hline HOMA-IR & $3.58 \pm 1.81$ & $3.20 \pm 1.81$ & $1.97 \pm 1.21$ & 0.244 & 0.001 & 0.007 \\
\hline CRP (mg/L) & $3.61 \pm 5.56$ & $3.66 \pm 5.21$ & $3.89 \pm 4.53$ & 0.928 & 0.997 & 0.985 \\
\hline Platelets $\left(\times 10^{9} / \mathrm{L}\right)$ & $204.71 \pm 64.30$ & $200.89 \pm 63.37$ & $193.1 \pm 66.78$ & 0.376 & 0.042 & 0.139 \\
\hline \multicolumn{7}{|l|}{ Histopathological Features } \\
\hline Significant inflammation ( $\mathrm{G} \geq 3, \mathrm{n}(\%))$ & 168 (39.44) & $234(40.00)$ & 71 (42.26) & 0.908 & 0.590 & 0.662 \\
\hline Advanced fibrosis ( $F \geq 3, n(\%))$ & $157(36.85)$ & $214(36.58)$ & $60(35.7 I)$ & 0.982 & 0.869 & 0.909 \\
\hline Moderate to severe steatosis $(S \geq 2, n(\%))$ & $216(50.70)$ & $260(44.44)$ & $52(30.95)$ & 0.057 & $<0.001$ & 0.002 \\
\hline
\end{tabular}

Notes: Categorical values are shown as $\mathrm{n}(\%)$. Continuous variables are shown as mean \pm standard deviation or median [interquartile range].

Abbreviations: MAFLD, metabolic associated fatty liver disease; NAFLD, nonalcoholic fatty liver disease; Non-MR-steatosis, non-metabolic risks NAFLD; BMI, body mass index; $\mathrm{HBsAg}$, hepatitis B surface antigen; ALT, alanine aminotransferase; AST, aspartate aminotransferase; GGT, $\gamma$-glutamyl transpeptidase; eGFR, estimated glomerular filtration rate; HDL-C, high-density lipoprotein cholesterol; LDL-C, low-density lipoprotein cholesterol; TG, triglyceride; TC, total cholesterol; VLDL-C, very low density lipoprotein cholesterol; FPG, fasting plasma glucose; HOMA-IR, Homeostasis model assessment-insulin resistance score; CRP, C-reactive protein.

NAFLD in a large biopsy-proven cohort. The main finding of our study is that there is no difference in steatosis and liver inflammation/fibrosis between MAFLD and NAFLD. Another important finding of our study is that a small group of patients with advanced fibrosis might be missed out by MAFLD criteria. These undiagnosed cases are lean and have fewer metabolic risks, but they still have severe inflammation and fibrosis in liver pathology. This specific subgroup of patients namely non-MR-steatosis exists regardless of the concomitant $\mathrm{HBV}$ infection.
The results of our study showed that non-MR-steatosis patients seemed to be much healthier from the angle of metabolic syndromes, for example, they were much younger than NAFLD and MAFLD, with the lowest BMI and normal metabolic profiles. This is not a surprising finding. However, their liver enzymes, including ALT, AST and GGT, were as high as those of MAFLD and NAFLD populations. The significant liver injury in non-MR-steatosis group was further evident by histopathological characteristics which showed that the extent of the inflammation and 
Table 2 Comparison Between MAFLD, NAFLD and Non-MR-Steatosis Groups in Non-HBV Population

\begin{tabular}{|c|c|c|c|c|c|c|}
\hline \multirow[t]{2}{*}{ Variables } & \multirow[t]{2}{*}{ MAFLD } & \multirow[t]{2}{*}{ NAFLD } & \multirow{2}{*}{$\begin{array}{l}\text { Non-MR- } \\
\text { Steatosis }\end{array}$} & \multicolumn{3}{|c|}{$\mathbf{P}$} \\
\hline & & & & $\begin{array}{c}\text { MAFLD } \\
\text { vs } \\
\text { NAFLD }\end{array}$ & $\begin{array}{l}\text { MAFLD vs } \\
\text { Non-MR- } \\
\text { Steatosis }\end{array}$ & $\begin{array}{l}\text { NAFLD vs } \\
\text { Non-MR- } \\
\text { Steatosis }\end{array}$ \\
\hline $\mathrm{N}$ & 60 & 74 & 18 & & & \\
\hline Age (years) & $41.62 \pm 15.26$ & $40.01 \pm|4.8|$ & $37.00 \pm 12.85$ & 0.559 & 0.288 & 0.488 \\
\hline Male, n (\%) & $36(60)$ & $48(64.86)$ & $13(72.22)$ & 0.690 & 0.507 & 0.753 \\
\hline BMI $\left(\mathrm{kg} / \mathrm{m}^{2}\right)$ & $25.3 \pm 3.35$ & $24.20 \pm 3.38$ & $20.97 \pm 1.25$ & 0.024 & $<0.001$ & $<0.001$ \\
\hline Type 2 diabetes, n (\%) & $17(28.33)$ & $16(21.62)$ & $0(0)$ & 0.487 & 0.008 & 0.035 \\
\hline Hypertension, n (\%) & $16(26.67)$ & $16(21.62)$ & I (5.56) & 0.633 & 0.100 & 0.177 \\
\hline TBIL ( $\mu \mathrm{molL})$ & $22.91 \pm 25.30$ & $20.84 \pm 22.97$ & $12.71 \pm 3.19$ & 0.830 & 0.288 & 0.316 \\
\hline Albumin $(g / L)$ & $44.11 \pm 6.34$ & $44.35 \pm 6.04$ & $44.26 \pm 4.34$ & 0.705 & 0.746 & 0.909 \\
\hline ALT (U/L) & $85(48,144.25)$ & $101(50,158)$ & $127(49.5,207.5)$ & 0.616 & 0.454 & 0.633 \\
\hline AST (U/L) & $46(33.25,66)$ & $48(32.5,78.5)$ & $61(34,80.5)$ & 0.729 & 0.638 & 0.785 \\
\hline GGT (U/L) & $103(59,230.25)$ & $105(53.5,204)$ & $105(49,139.5)$ & 0.708 & 0.208 & 0.300 \\
\hline Creatinine ( $\mu \mathrm{molL})$ & $67.32 \pm 14.53$ & $67.56 \pm 14.95$ & $65.46 \pm 17.06$ & 0.891 & 0.743 & 0.712 \\
\hline eGFR (mL/min) & $136.3 \pm 38.63$ & $136.44 \pm 39.69$ & $142.19 \pm 43.27$ & 0.972 & 0.694 & 0.690 \\
\hline Uric acid ( $\mu$ molL) & $356.67 \pm 76.29$ & $354.23 \pm 80.54$ & $337.93 \pm 100.08$ & 0.711 & 0.190 & 0.256 \\
\hline $\mathrm{TC}(\mathrm{mmol} / \mathrm{L})$ & $4.91 \pm 1.08$ & $4.97 \pm 1.07$ & $4.98 \pm 0.96$ & 0.688 & 0.629 & 0.811 \\
\hline TG (mmol/L) & $2.12 \pm 2.26$ & $1.97 \pm 2.05$ & $1.40 \pm 0.29$ & 0.518 & 0.091 & 0.216 \\
\hline LDL-C (mmol/L) & $2.91 \pm 1.04$ & $2.95 \pm 1.01$ & $3.08 \pm 0.69$ & 0.790 & 0.581 & 0.733 \\
\hline HDL-C (mmol/L) & $1.13 \pm 0.35$ & $1.18 \pm 0.37$ & $1.26 \pm 0.42$ & 0.616 & 0.458 & 0.675 \\
\hline VLDL-C (mmol/L) & $0.65 \pm 0.51$ & $0.61 \pm 0.49$ & $0.35 \pm 0.16$ & 0.418 & 0.006 & 0.023 \\
\hline $\mathrm{FPG}(\mathrm{mmol} / \mathrm{L})$ & $5.42 \pm 1.03$ & $5.27 \pm 0.95$ & $4.88 \pm 0.44$ & 0.289 & 0.013 & 0.062 \\
\hline Platelets $\left(\times 10^{9} / \mathrm{L}\right)$ & $240.27 \pm 77.17$ & $237.01 \pm 74.12$ & $224.86 \pm 47.21$ & 0.754 & 0.440 & 0.540 \\
\hline \multicolumn{7}{|l|}{ Histopathological Features } \\
\hline Significant inflammation ( $\mathrm{G} \geq 3, \mathrm{n}(\%))$ & II (I8.33) & $12(16.22)$ & $2(11.11)$ & 0.926 & 0.721 & 0.729 \\
\hline Advanced fibrosis ( $\mathrm{F} \geq 3, \mathrm{n}(\%))$ & $9(15.00)$ & $10(|3.5|)$ & $2(11.11)$ & 1.000 & 1.000 & 1.000 \\
\hline Moderate to severe steatosis $(S \geq 2, n(\%))$ & $49(81.67)$ & $58(78.38)$ & $12(66.67)$ & 0.798 & 0.201 & 0.357 \\
\hline NAS score & $4.38 \pm 1.55$ & $4.27 \pm 1.59$ & $3.89 \pm 1.68$ & 0.712 & 0.338 & 0.457 \\
\hline
\end{tabular}

Notes: Categorical values are shown as $\mathrm{n}(\%)$. Continuous variables are shown as mean \pm standard deviation or median [interquartile range].

Abbreviations: MAFLD, metabolic associated fatty liver disease; NAFLD, nonalcoholic fatty liver disease; Non-MR-steatosis, non-metabolic risks NAFLD; BMI, body mass index; $\mathrm{HBsAg}$, hepatitis B surface antigen; ALT, alanine aminotransferase; AST, aspartate aminotransferase; GGT, $\gamma$-glutamyl transpeptidase; eGFR, estimated glomerular filtration rate; HDL-C, high-density lipoprotein cholesterol; LDL-C, low-density lipoprotein cholesterol; TG, triglyceride; TC, total cholesterol; VLDL-C, very low density lipoprotein cholesterol; FPG, fasting plasma glucose.

the degree of fibrosis was not different from the other two groups. The result remained consistent in HBV and nonHBV subgroups in this cohort, indicating the hepatic steatosis itself may lead to liver injury. Similarly, our previous study based on the National Health and Nutrition Examination Survey dataset also showed that, a group of steatotic patients who had advanced fibrosis assessed by non-invasive scores will be undiagnosed by MAFLD criteria. $^{12}$ Taken together, these results strongly suggest the novel MAFLD criteria may miss out on some steatotic patients with extensive liver injury.

The clinical, biochemical and histopathological characteristics of non-MR-steatosis are similar to that of lean NAFLD, which is known to have normal BMI and a very low proportion of type 2 diabetes $(0.6 \%)$, hypertension
$(1.8 \%)$, metabolic syndrome $(1.4 \%)$, dyslipidemia $(2.8 \%)$, or obesity $(2.0 \%){ }^{20}$ Patients with lean NAFLD still at high risk of having advanced grade steatohepatitis or fibrosis. ${ }^{21}$ The long-term follow-up studies had illustrated considerable all-cause mortality, liver-related mortality, ${ }^{22}$ and an increased risk of advanced liver disease $^{23}$ in the lean NAFLD population. All these studies underline the importance of the identification of patients with lean NAFLD as they may have an equivalent risk of disease progression and liver-related mortality. However, some lean NAFLD may have no metabolic dysfunction thus being missed diagnosed by MAFLD. In the light of MAFLD, there is no place for this specific group and this will eventually lead to missed opportunities for meaningful interventions in this group of patients. 
Table 3 Comparison Between MAFLD, NAFLD and Non-MR-Steatosis Groups in HBV-Infected Population

\begin{tabular}{|c|c|c|c|c|c|c|}
\hline \multirow[t]{2}{*}{ Variables } & \multirow[t]{2}{*}{ MAFLD } & \multirow[t]{2}{*}{ NAFLD } & \multirow{2}{*}{$\begin{array}{l}\text { Non-MR- } \\
\text { Steatosis }\end{array}$} & \multicolumn{3}{|c|}{$P$} \\
\hline & & & & $\begin{array}{c}\text { MAFLD } \\
\text { vs } \\
\text { NAFLD }\end{array}$ & $\begin{array}{l}\text { MAFLD vs } \\
\text { Non-MR- } \\
\text { Steatosis }\end{array}$ & $\begin{array}{l}\text { NAFLD vs } \\
\text { Non-MR- } \\
\text { Steatosis }\end{array}$ \\
\hline$N$ & 366 & 511 & 150 & & & \\
\hline Age (years) & $40.19 \pm 10.10$ & $39.32 \pm 10.47$ & $37.06 \pm 11.05$ & 0.208 & 0.001 & 0.014 \\
\hline Male, n (\%) & $325(88.80)$ & $44 \mid(86.3)$ & $120(80)$ & 0.320 & 0.013 & 0.078 \\
\hline BMI $\left(\mathrm{kg} / \mathrm{m}^{2}\right)$ & $25.54 \pm 2.53$ & $24.17 \pm 3.16$ & $20.76 \pm 1.58$ & $<0.001$ & $<0.001$ & $<0.001$ \\
\hline Waist circumference $(\mathrm{cm})$ & $84.28 \pm 7.43$ & $84.83 \pm 6.87$ & $86.10 \pm 5.53$ & 0.777 & 0.524 & 0.640 \\
\hline Type 2 diabetes, $\mathrm{n}(\%)$ & $66(18.03)$ & $65(12.72)$ & $0(0)$ & 0.037 & $<0.001$ & $<0.001$ \\
\hline Hypertension, n (\%) & $26(7.10)$ & $29(5.68)$ & $3(2.00)$ & 0.472 & 0.038 & 0.104 \\
\hline Log HBVDNA (IU/mL) & $5.06 \pm 2.14$ & $5.13 \pm 2.11$ & $5.29 \pm 2.01$ & 0.613 & 0.291 & 0.463 \\
\hline HBeAg positive, $n$ (\%) & $163(44.54)$ & 235 (45.99) & $73(48.67)$ & 0.670 & 0.392 & 0.563 \\
\hline TBIL ( $\mu \mathrm{molL})$ & $|7.7| \pm 20.75$ & $17.78 \pm 21.17$ & $17.85 \pm 21.94$ & 0.993 & 0.984 & 0.989 \\
\hline Albumin $(g / L)$ & $42.33 \pm 4.64$ & $42.26 \pm 4.85$ & $42.05 \pm 5.29$ & 0.724 & 0.326 & 0.444 \\
\hline ALT (U/L) & $62(40,142)$ & $65(39.5,139)$ & $73(39.5,129)$ & 0.959 & 0.738 & 0.755 \\
\hline AST (U/L) & $42(30,72)$ & $42(30,77)$ & $44(32,81.5)$ & 0.778 & 0.314 & 0.401 \\
\hline GGT (U/L) & $48(30,81)$ & $46(29,75)$ & $40(27,68)$ & 0.204 & 0.007 & 0.056 \\
\hline Creatinine $(\mu \mathrm{molL})$ & $74.41 \pm 13.44$ & $74.65 \pm 18.22$ & $74.90 \pm 26.59$ & 0.863 & 0.524 & 0.589 \\
\hline eGFR (mL/min) & $118.15 \pm 30.53$ & $119.76 \pm 36.18$ & $|24.5| \pm 47.21$ & 0.657 & 0.179 & 0.281 \\
\hline Uric acid ( $\mu \mathrm{molL})$ & $371.38 \pm 83.19$ & $363.75 \pm 83.36$ & $345.60 \pm 80.09$ & 0.233 & 0.005 & 0.044 \\
\hline $\mathrm{TC}(\mathrm{mmol} / \mathrm{L})$ & $4.74 \pm 1.14$ & $4.71 \pm 1.08$ & $4.6 \pm 0.86$ & 0.676 & 0.289 & 0.424 \\
\hline $\mathrm{TG}(\mathrm{mmol} / \mathrm{L})$ & $1.61 \pm 0.96$ & $1.44 \pm 0.88$ & $1.03 \pm 0.43$ & $<0.001$ & $<0.001$ & $<0.001$ \\
\hline LDL-C (mmol/L) & $2.89 \pm 0.95$ & $2.83 \pm 0.93$ & $2.65 \pm 0.83$ & 0.376 & 0.023 & 0.083 \\
\hline HDL-C (mmol/L) & $1.12 \pm 0.3$ & $1.20 \pm 0.34$ & $1.37 \pm 0.38$ & 0.002 & $<0.001$ & $<0.001$ \\
\hline VLDL-C (mmol/L) & $0.49 \pm 0.35$ & $0.44 \pm 0.32$ & $0.28 \pm 0.15$ & 0.005 & $<0.001$ & $<0.001$ \\
\hline FPG (mmol/L) & $5.32 \pm 1.47$ & $5.12 \pm 1.27$ & $4.68 \pm 0.55$ & 0.011 & $<0.001$ & $<0.001$ \\
\hline HOMA-IR & $3.53 \pm 1.84$ & $3.15 \pm 1.82$ & $1.97 \pm 1.21$ & 0.252 & 0.001 & 0.011 \\
\hline CRP (mg/L) & $2.78 \pm 5.03$ & $3.01 \pm 4.71$ & $3.40 \pm 4.53$ & 0.587 & 0.711 & 0.758 \\
\hline Platelets $\left(\times 10^{9} / \mathrm{L}\right)$ & $198.87 \pm 60.06$ & $195.76 \pm 60.03$ & $189.75 \pm 67.79$ & 0.467 & 0.071 & 0.180 \\
\hline \multicolumn{7}{|l|}{ Histopathological Features } \\
\hline Significant inflammation $(\mathrm{G} \geq 3, \mathrm{n}(\%))$ & $157(42.90)$ & $222(43.44)$ & $69(46.00)$ & 0.926 & 0.584 & 0.645 \\
\hline Advanced fibrosis ( $F \geq 3, n(\%))$ & $148(40.44)$ & $204(39.92)$ & $58(38.67)$ & 0.933 & 0.784 & 0.856 \\
\hline Moderate to severe steatosis $(S \geq 2, n(\%))$ & $167(45.63)$ & $202(39.53)$ & $40(26.67)$ & 0.083 & $<0.001$ & 0.005 \\
\hline
\end{tabular}

Notes: Categorical values are shown as $\mathrm{n}(\%)$. Continuous variables are shown as mean \pm standard deviation or median [interquartile range].

Abbreviations: MAFLD, metabolic associated fatty liver disease; NAFLD, nonalcoholic fatty liver disease; Non-MR-steatosis, non-metabolic risks NAFLD; BMI, body mass index; HBsAg, hepatitis B surface antigen; ALT, alanine aminotransferase; AST, aspartate aminotransferase; GGT, $\gamma$-glutamyl transpeptidase; eGFR, estimated glomerular filtration rate; HDL-C, high-density lipoprotein cholesterol; LDL-C, low-density lipoprotein cholesterol; TG, triglyceride; TC, total cholesterol; VLDL-C, very low density lipoprotein cholesterol; FPG, fasting plasma glucose; HOMA-IR, Homeostasis model assessment-insulin resistance score; CRP, C-reactive protein.

It is worth noting that most patients included in our study were having elevated liver enzymes and were clinically suspected to have liver cell injury which was further evaluated with a liver biopsy. Although serum ALT and AST are not sensitive biomarkers for hepatocellular injury in patients with hepatic steatosis, ${ }^{24}$ the results indicated the role of elevated liver enzymes for diagnostic criteria of MAFLD should also be considered.

The strength of this study is that to our knowledge this is the first study to evaluate the diagnostic criteria of MAFLD based on liver histopathology. Our study should however be interpreted in light of some limitations. First, the study cohort is from a single center with a high proportion of $\mathrm{HBV}$ and low BMI, which is a common occurrence in Asian countries, ${ }^{25,26}$ limiting the generality of our results to the western population. But at least the results of this study apply to a subset of MAFLD namely "HBV-MAFLD", which is the most important subtype of MAFLD in clinical practice in Asia. Second, compared with our previous population-based study, ${ }^{11}$ this study only included highly selected patients who underwent liver biopsy, however, it is also fairly common in clinical practice where liver biopsy is only performed in those who 
were highly suspected to have a liver injury. Third, there were only 82 cases in the non-HBV subgroup, which might lead to type II errors in statistical analysis. But in line with our previous study in which the NHANES database was used, the fibrosis assessed by non-invasive models was also significant in some non-MR-steatosis cases. ${ }^{12}$ Nevertheless, more evidence is needed to verify this conclusion. At last, we did not conclude patients with excessive alcohol consumption because those patients seldom received liver biopsies in our department. In another study, we showed that MAFLD with excessive alcohol consumption has less number of metabolic conditions but equally severe liver injury. ${ }^{27}$ Moreover, in patients with MAFLD, even mild alcohol consumption is associated with worsening of hepatic fibrosis measures. ${ }^{28,29}$ Thus for patients with alcohol consumption, more aggressive management are needed.

In conclusion, MAFLD criteria may overlook a proportion of patients with non-metabolic related steatohepatitis and significant fibrosis.

\section{Abbreviations}

FLD, fatty liver disease; MAFLD, metabolic associated fatty liver disease; NAFLD, nonalcoholic fatty liver disease; NonMR-steatosis, Non-metabolic risks NAFLD; NAS, NAFLD activity score; BMI, body mass index; HBsAg, hepatitis B surface antigen; HBV, hepatitis B virus; ALT, alanine aminotransferase; AST, aspartate aminotransferase; GGT, $\gamma$ glutamyl transpeptidase; eGFR, estimated glomerular filtration rate; HDL-C, high-density lipoprotein cholesterol; LDL-C, low-density lipoprotein cholesterol; TG, triglyceride; TC, total cholesterol; VLDL-C, very low-density lipoprotein cholesterol; FPG, fasting plasma glucose; HOMA-IR, homeostasis model assessment-insulin resistance score; CRP, C-reactive protein.

\section{Ethics Approval and Informed Consent}

An informed consent was obtained from each participant on admission to use their medical data anonymously. The ethics approval was approved by the First Affiliated Hospital, Fujian Medical University Institution Review Board ([2015]084-1). This study conforms to the principles outlined in the Declaration of Helsinki.

\section{Author Contributions}

Jiaofeng Huang and Wenjuan Xue, share first authorship. Study concept and design: Jiaofeng Huang and Su Lin
Acquisition, cleaning of data: Wenjuan Xue and Mingfang Wang

Drafting of the manuscript: Su Lin and Jiaofeng Huang Critical revision: Rahul Kumar, Yueyong Zhu, Medha Singh and Yinlian $\mathrm{Wu}$

Statistical analysis: Jiaofeng Huang and Wenjuan Xue Study supervision: Su Lin

All authors made a significant contribution to the work reported, whether that is in the conception, study design, execution, acquisition of data, analysis and interpretation, or in all these areas; took part in drafting, revising or critically reviewing the article; gave final approval of the version to be published; have agreed on the journal to which the article has been submitted; and agree to be accountable for all aspects of the work.

\section{Funding}

This research is supported by Qingzhong Medical Science Research Fund (B17344) and Startup Fund for scientific research, Fujian Medical University (2018QH1047).

\section{Disclosure}

The authors declare that they have no competing interests in this work.

\section{References}

1. Eslam M, Sanyal AJ, George J. MAFLD: a consensus-driven proposed nomenclature for metabolic associated fatty liver disease. Gastroenterology. 2020;158(7):1999-2014.e1991. doi:10.1053/j. gastro.2019.11.312

2. Valenti L, Pelusi S. Redefining fatty liver disease classification in 2020. Liver Int. 2020;40(5):1016-1017. doi:10.1111/liv.14430

3. EASL E. EASL-EASD-EASO Clinical Practice Guidelines for the management of non-alcoholic fatty liver disease. $J$ Hepatol. 2016;64 (6):1388-1402. doi:10.1016/j.jhep.2015.11.004

4. Fouad Y, Waked I, Bollipo S, et al. What's in a name? Renaming 'NAFLD' to 'MAFLD'. Liver Int. 2020;40(6):1254-1261. doi:10.1111/liv.14478

5. Eslam M, Newsome PN, Sarin SK, et al. A new definition for metabolic dysfunction-associated fatty liver disease: an international expert consensus statement. J Hepatol. 2020;73(1):202-209. doi:10.1016/j. jhep.2020.03.039

6. Wai-Sun Wong V, Lai-Hung Wong G, Woo J, et al. Impact of the new definition of metabolic associated fatty liver disease on the epidemiology of the disease. Clin Gastroenterol Hepatol. 2020. doi:10.1016/j. cgh.2020.10.046

7. Zheng KI, Sun DQ, Jin Y, et al. Clinical utility of the MAFLD definition. $J$ Hepatol. 2021;74(4):989-991. doi:10.1016/j. jhep.2020.12.016

8. Sun DQ, Jin Y, Wang TY, et al. MAFLD and risk of CKD. Metabolism. 2021;115:154433. doi:10.1016/j.metabol.2020.154433

9. Majumdar A, Tsochatzis E. Changing trends of liver transplantation and mortality from non-alcoholic fatty liver disease. Metabolism. 2020;111:154291. doi:10.1016/j.metabol.2020.154291 
10. Cotter T, Dong L, Holmen J, et al. Nonalcoholic fatty liver disease: impact on healthcare resource utilization, liver transplantation and mortality in a large, integrated healthcare system. J Gastroenterol. 2020;55(7):722-730. doi:10.1007/s00535-020-01684-w

11. Lin S, Huang J, Wang M, et al. Comparison of MAFLD and NAFLD diagnostic criteria in real world. Liver Int. 2020;40(9):2082-2089. doi:10.1111/liv.14548

12. Huang J, Kumar R, Wang M, et al. MAFLD criteria overlooks a number of patients with severe steatosis: is it clinically relevant? J Hepatol. 2020;73(5):1265-1267. doi:10.1016/j.jhep.2020.06.016

13. Wu Y, Kumar R, Huang J, et al. FIB-4 cut-off should be re-evaluated in patients with metabolic associated fatty liver disease (MAFLD). J Hepatol. 2021;74(1):247-248. doi:10.1016/j.jhep.2020.06.040

14. Wang FS, Fan JG, Zhang Z, et al. The global burden of liver disease: the major impact of China. Hepatology. 2014;60(6):2099-2108. doi:10.1002/hep. 27406

15. Bell S, Lau A, Thompson A, et al. Chronic hepatitis B: recommendations for therapy based on the natural history of disease in Australian patients. $J$ Clin Virol. 2005;32(2):122-127. doi:10.1016/j. jcv.2004.10.009

16. Kleiner DE, Brunt EM, Van Natta M, et al. Design and validation of a histological scoring system for nonalcoholic fatty liver disease. Hepatology. 2005;41(6):1313-1321. doi:10.1002/hep.20701

17. Brunt EM. Grading and staging the histopathological lesions of chronic hepatitis: the Knodell histology activity index and beyond. Hepatology. 2000;31(1):241-246. doi:10.1002/hep.510310136

18. Scheuer PJ. Classification of chronic viral hepatitis: a need for reassessment. J Hepatol. 1991;13(3):372-374. doi:10.1016/01688278(91)90084-O

19. Hong M, Zhang R, Chen G, et al. Liver stiffness measurement-based scoring system for significant inflammation related to chronic hepatitis B. PLoS One. 2014;9(10):e111641. doi:10.1371/journal. pone.0111641

20. Lu FB, Zheng KI, Rios RS, et al. Global epidemiology of lean non-alcoholic fatty liver disease: a systematic review and meta-analysis. J Gastroenterol Hepatol. 2020;35(12):2041-2050. doi:10.1111/jgh. 15156
21. Eslam M, Fan JG, Mendez-Sanchez N. Non-alcoholic fatty liver disease in non-obese individuals: the impact of metabolic health. Lancet Gastroenterol Hepatol. 2020;5(8):713-715. doi:10.1016/ S2468-1253(20)30090-X

22. Ye Q, Zou B, Yeo YH, et al. Global prevalence, incidence, and outcomes of non-obese or lean non-alcoholic fatty liver disease: a systematic review and meta-analysis. Lancet Gastroenterol Hepatol. 2020;5(8):739-752. doi:10.1016/S2468-1253(20)30077-7

23. Hagström H, Nasr P, Ekstedt M, et al. Risk for development of severe liver disease in lean patients with nonalcoholic fatty liver disease: a long-term follow-up study. Hepatol Commun. 2018;2(1):48-57. doi:10.1002/hep4.1124

24. Francque S, Laleman W, Verbeke L, et al. Increased intrahepatic resistance in severe steatosis: endothelial dysfunction, vasoconstrictor overproduction and altered microvascular architecture. Lab Invest. 2012;92(10):1428-1439. doi:10.1038/labinvest.2012.103

25. WHO Expert Consultation. Appropriate body-mass index for Asian populations and its implications for policy and intervention strategies. Lancet. 2004;363(9403):157-163. doi:10.1016/S0140-6736(03) 15268-3

26. Huang J, Jing M, Wang C, et al. The impact of hepatitis B virus infection status on the prevalence of nonalcoholic fatty liver disease: a population-based study. J Med Virol. 2020;92(8):1191-1197. doi:10.1002/jmv.25621

27. Huang J, Ou W, Wang M, et al. MAFLD criteria guide the subtyping of patients with fatty liver disease. Risk Manag Healthc Policy. 2021;14:491-501. doi:10.2147/RMHP.S285880

28. Yamamura S, Eslam M, Kawaguchi T, et al. MAFLD identifies patients with significant hepatic fibrosis better than NAFLD. Liver Int. 2020;40(12):3018-3030. doi:10.1111/liv.14675

29. Wang MF, Wan B, Wu YL, et al. Clinic-pathological features of metabolic associated fatty liver disease with hepatitis B virus infection. World J Gastroenterol. 2021;27(4):336-344. doi:10.3748/ wjg.v27.i4.336

Diabetes, Metabolic Syndrome and Obesity: Targets and Therapy

Dovepress

\section{Publish your work in this journal}

Diabetes, Metabolic Syndrome and Obesity: Targets and Therapy is an international, peer-reviewed open-access journal committed to the rapid publication of the latest laboratory and clinical findings in the fields of diabetes, metabolic syndrome and obesity research. Original research, review, case reports, hypothesis formation, expert opinion and commentaries are all considered for publication. The manuscript management system is completely online and includes a very quick and fair peer-review system, which is all easy to use. Visit http://www.dovepress.com/testimonials.php to read real quotes from published authors.

Submit your manuscript here: https://www.dovepress.com/diabetes-metabolic-syndrome-and-obesity-targets-and-therapy-journal 